\title{
B-BRANES AND THE DERIVED CATEGORY
}

\author{
SHELDON KATZ \\ Departments of Mathematics and Physics \\ University of Illinois at Urbana-Champaign \\ Urbana, IL 61801 \\ Email:katz@math.uiuc.edu
}

\begin{abstract}
By a direct CFT computation, the spectrum of the topological B-model is compared to Ext groups of sheaves. A match can only be made if abstract vector bundles on holomorphic submanifolds are twisted by the canonical Spin ${ }^{c}$ structure of its support in describing physical branes.
\end{abstract}

\section{Introduction.}

In recent years, there has been much fruitful interplay between physics and geometry through string theory. Many questions in physics correspond to questions in pure geometry. The motivations in physics and geometry are often of a completely different nature, so that finding the precise dictionary between the two areas is key to making fundamental progress.

The spectrum of the closed topological string is well understood ${ }^{15}$, and the correlation functions can be computed. In this talk, I outline a similar description for the open string sector of the topological B-model on a compact Kähler manifold $X$. The first task is to understand the boundary conditions, which are to be identified with the derived category in wellknown ways ${ }^{10,12,5,1}$. The simplest case arises from a holomorphic submanifold $i: S \hookrightarrow X$ and holomorphic vector bundle $E$ on $X$, so that a D-brane is wrapped on $S$ with gauge bundle $E$. This data can be identified with the sheaf $i_{*} E$ of $\mathcal{O}_{X}$ modules, which is not a bundle unless $S=X$. In any case, $i_{*} E$ is an object of the derived category of $X$. More general objects of the derived category can be identified with boundary conditions as well.

This pleasing identification of geometric objects with boundary conditions is complicated by worldsheet anomalies, which can be eliminated by twisting the gauge bundle by a $\operatorname{Spin}^{c}$ structure $^{6}$. A canonical choice is given by the canonical $\operatorname{Spin}^{c}$ structure on $X$. This corresponds to modifying the gauge bundle $E$ to become, somewhat imprecisely, $E \otimes K_{S}^{-1 / 2}$. With this 
choice, the open string spectrum matches Ext groups exactly ${ }^{9}$. With any other twisting or no twisting at all, the spectrum can fail to match Ext groups. So the identification of objects of the derived category with branes is a bit more subtle than one would like.

In this talk, I give the basic examples illustrating how to compute the spectrum directly in BCFT and how the results are only sensible when the anomaly is included in the analysis. More details are available elsewhere ${ }^{9,13}$, as are generalizations to orbifolds ${ }^{8}$, flat B-field backgrounds ${ }^{3}$, and D-branes wrapping nonreduced schemes ${ }^{4,14}$.

\section{Closed string B model}

I start by reviewing the topological $B$-model on a Calabi-Yau threefold $X$. Locally $X$ has three complex coordinates $\left(\phi^{1}, \phi^{2}, \phi^{3}\right)$ which can be identified with bosonic fields on the worldsheet. A nowhere vanishing antisymmetric holomorphic tensor $\Omega=\Omega_{i j k}$ is required to soak up fermion zero modes. This condition is satisfied for a Calabi-Yau threefold. The topological Bmodel can be compared to physical strings in $10 \mathrm{D} M^{4} \times X$; I do not do that here but make some brief comments at the end.

Tensors on $X$ have holomorphic indices $i$ and antiholomorphic indices $\bar{i}$. These are associated to the holomorphic and antiholomorphic tangent bundles of $X$ which are global objects, i.e.

$$
\begin{aligned}
\rho^{i} & \in T^{1,0}(X) \\
\rho^{\bar{i}} & \in T^{0,1}(X)
\end{aligned}
$$

with $\operatorname{det}\left(T^{1,0}(X)\right)$ a trivial bundle, required by fermion zero modes coupling to $T^{1,0}(X)$ as mentioned above.

The worldsheet is a Riemann surface $\Sigma$ with local complex coordinate $z$. Covariant tensors split into holomorphic and antiholomorphic pieces. The holomorphic part is called the canonical bundle and is denoted by $K_{\Sigma}=$ $\left(T^{1,0}(\Sigma)\right)^{*}$. The antiholomorphic part is denoted by $\bar{K}_{\Sigma}=\left(T^{0,1}(\Sigma)\right)^{*}$

The fields in the topological string combine the tensor structures on $\Sigma$ and $X$. So the fields in the theory are:

$$
\begin{array}{lll} 
& \multicolumn{3}{c}{\phi^{i}(z)} \\
\psi_{ \pm}^{\bar{\tau}}(z) & \in & \Gamma\left(\phi^{*} T^{0,1} X\right), \\
\psi_{+}^{i}(z) & \in & \Gamma\left(K \otimes \phi^{*} T^{1,0} X\right), \\
\psi_{-}^{i}(z) & \in & \Gamma\left(\bar{K} \otimes \phi^{*} T^{1,0} X\right)
\end{array}
$$


The $\psi$ fields anticommute with each other, and the $\phi$ are bosonic. The bulk B model Lagrangian is

$$
\begin{gathered}
\frac{1}{2} g_{i \bar{\jmath}} \partial \phi^{i} \bar{\partial} \phi^{\bar{\jmath}}+\frac{1}{2} g_{i \bar{\jmath}} \partial \phi^{\bar{\jmath}} \bar{\partial} \phi^{i}+i g_{i \bar{\jmath}} \psi_{-}^{\bar{\jmath}} D_{z} \psi_{-}^{i}+ \\
i g_{i \bar{\jmath}} \psi_{+}^{\bar{\jmath}} D_{\bar{z}} \psi_{+}^{i}+R_{i \bar{\imath} j \bar{\jmath}} \psi_{+}^{i} \psi_{+}^{\bar{\imath}} \psi_{-}^{j} \psi_{-}^{\bar{\jmath}}
\end{gathered}
$$

with BRST transformations

$$
\begin{aligned}
\delta \phi^{i} & =0, \\
\delta \phi^{\bar{\imath}} & =i \alpha\left(\psi_{+}^{\bar{\tau}}+\psi_{-}^{\bar{\imath}}\right), \\
\delta \psi_{+}^{i} & =-\alpha \partial \phi^{i}, \\
\delta \psi_{+}^{\bar{\tau}} & =-i \alpha \psi_{-}^{\bar{\jmath}} \Gamma_{\bar{\jmath} m}^{\bar{\tau}} \psi_{+}^{\bar{m}}, \\
\delta \psi_{-}^{i} & =-\alpha \bar{\partial} \phi^{i}, \\
\delta \psi_{-}^{\bar{\tau}} & =-i \alpha \psi_{+}^{\bar{\jmath}} \Gamma^{\bar{\imath}} \psi_{-}^{\bar{m}} .
\end{aligned}
$$

The BRST symmetry satisfies $\delta^{2}=0$. Consideration is restricted to BRST-closed operators, $\delta F=0$. Addition of a BRST-exact operator does not alter correlation functions

$$
F \sim F+\delta G,
$$

so the spectrum is described by BRST cohomology

$$
H_{\mathrm{BRST}}^{*}=\frac{\{\mathrm{BRST}-\text { closed operators }\}}{\{\mathrm{BRST}-\text { exact operators }\}} .
$$

It is convenient to change variables and define

$$
\begin{aligned}
\eta^{\bar{\tau}} & =\psi_{+}^{\bar{\tau}}+\psi_{-}^{\bar{\tau}}, \\
\theta_{i} & =g_{i \bar{\jmath}}\left(\psi_{+}^{\bar{\jmath}}-\psi_{-}^{\bar{\jmath}}\right), \\
\rho_{z}^{i} & =\psi_{+}^{i}, \\
\rho_{\bar{z}}^{i} & =\psi_{-}^{i},
\end{aligned}
$$

A vertex operator is of the form

$$
b_{\bar{\imath}_{1} \cdots \bar{\tau}_{n}}^{j_{1} \cdots j_{m}}\left(\phi_{0}\right) \eta^{\bar{\imath}_{1}} \cdots \eta^{\bar{z}_{n}} \theta_{j_{1}} \cdots \theta_{j_{m}}
$$

This operator can be identified with a tensor field on $X$ :

$$
b_{\bar{\imath}_{1} \cdots \bar{i}_{n}}^{j_{1} \cdots j_{m}}(\phi) d \phi^{\bar{i}_{1}} \wedge \ldots \wedge d \phi^{\bar{i}_{n}} \otimes \frac{\partial}{\partial \phi^{j_{1}}} \wedge \ldots \wedge \frac{\partial}{\partial \phi^{j_{m}}}
$$

where $\wedge$ denotes a completely antisymmetrized product.

Under this identification, BRST-cohomology is identified with the Dolbeault cohomology of $\Lambda T^{1,0}(X)$, an important geometric invariant of the holomorphic structure of $X$. 


$$
H^{q}\left(\Lambda^{p} T^{1,0}(X)\right)=\frac{\{\bar{\partial}-\operatorname{closed}(0, q) \text { forms }\}}{\{\bar{\partial}-\operatorname{exact}(0, q) \text { forms }\}}
$$

where the forms take values in $\Lambda^{p} T^{1,0}(X)$. Here $\bar{\partial}$ is the antiholomorphic differential, e.g.

$$
\bar{\partial} f=\frac{\partial f}{\partial \bar{\phi}^{i}} d \bar{\phi}^{i} .
$$

Among these Dolbeault cohomology classes, $H^{1}\left(T^{1,0}(X)\right)$ parametrizes the holomorphic structure.

Correlation functions of the associated vertex operators are important. For example, three point correlators of operators associated to $H^{1}\left(T^{1,0}(X)\right)$

$$
\left\langle A_{\bar{j}_{1}}^{i_{1}} \theta_{i_{1}} \eta^{\bar{j}_{1}}, A_{\bar{j}_{2}}^{i_{2}} \theta_{i_{2}} \eta^{\bar{j}_{2}}, A_{\bar{j}_{3}}^{i_{3}} \theta_{i_{3}} \eta^{\bar{j}_{3}}\right\rangle
$$

are identified with the Yukawa couplings in the gauge sector of the heterotic string.

This is the kind of description to be extended to the open string.

\section{Open string B-model}

In extending the previous analysis to open string the first step is to find the boundary conditions. The simplest supersymmetric B-branes are described by a holomorphic submanifold $S \subset X$ together with a gauge field $A_{i}$ on $S$. The coordinates on $S$ are Neumann directions while coordinates transverse to $S$ are Dirichlet directions. Using the gauge field, introduce the usual boundary term in the action

$$
\int_{\partial \Sigma} A_{i} \frac{\partial \phi^{i}}{\partial t}+\ldots
$$

Along Neumann directions, the boundary conditions are

$$
\left.\psi_{+}^{i}\right|_{\partial \Sigma}=\left.\psi_{-}^{i}\right|_{\partial \Sigma}, \quad \theta_{i}=\left(\operatorname{Tr} F_{i \bar{\jmath}}\right) \eta^{\bar{\jmath}},
$$

$F$ being the field strength, while along Dirichlet directions, the boundary conditions are

$$
\left.\psi_{+}^{i}\right|_{\partial \Sigma}=-\left.\psi_{-}^{i}\right|_{\partial \Sigma}, \quad \eta^{\bar{\imath}}=0
$$


Some care is needed in interpreting the intrinsic meaning of a Dirichlet direction, since the tangent bundle of $X$ along $S$ has a $C^{\infty}$ splitting into the sum of the tangent bundle to $S$ and the normal bundle to $S$ in $X$, i.e. $\left.T X\right|_{S} \simeq T S \oplus N_{S / X}$, but there need not be a holomorphic splitting.

The vertex operators are

$$
b_{\bar{\imath}_{1} \cdots \bar{\tau}_{n}}^{\alpha \beta j_{m} \cdots j_{m}}\left(\phi_{0}\right) \eta^{\overline{1}_{1}} \cdots \eta^{\bar{\tau}_{n}} \theta_{j_{1}} \cdots \theta_{j_{m}}
$$

where the $\bar{i}$ are indices on $S$ and the $\theta_{j}$ are constrained by the boundary conditions. Also, $\alpha, \beta$ are gauge degrees of freedom (Chan-Paton indices).

The immediate goal is to evaluate the BRST cohomology and identify it with a geometric object. Mathematically, a novel complex of differential forms has been defined, the novelty being the boundary conditions, and the problem is to compute its cohomology. The answer is expected to match Ext groups of objects in the derived category of algebraic geometry. This does check, with some surprises along the way.

Now $U(n)$ gauge fields live in rank $n$ vector bundles $E$ on $S$. Locally on $S, E$ takes the form $U_{\alpha} \times \mathbf{C}^{n}$ for $U_{\alpha} \subset S$. Vector bundles are glued together over intersections $U_{\alpha} \cap U_{\beta}$ via $r \times r$ matrices $g_{\alpha \beta}$ acting on $\mathbf{C}^{r}$ by multiplication. Sections of bundles are identified with local $\mathbf{C}^{r}$-valued functions $s_{\alpha}$ on each $U_{\alpha}$ satisfying

$$
s_{\alpha}=g_{\alpha \beta} s_{\beta} .
$$

The sections of $E$ form an important object of geometry called a coherent sheaf on $X$. This means that sections $s_{\alpha}$ of $E$ can be multiplied by holomorphic functions $f$ on $X$, and that the result only depends on the restriction of such functions to $S$ (coherence is a technical finiteness condition which is automatically satisfied here). Explicitly the multiplication is simply the identity $\left.f\right|_{S} \cdot s_{\alpha}=g_{\alpha \beta}\left(\left(\left.f\right|_{S}\right) \cdot s_{\beta}\right)$ obtained from (1) after multiplication by $\left.f\right|_{S}$. The resulting sheaf is denoted $i_{*} E$ where $i: S \hookrightarrow X$ is the inclusion.

Coherent sheaves form the heart of a t-structure on the derived category of $X$. From this heart, all objects of the derived category can be built by shifts and extensions. The heart, shifts, and extensions model branes, antibranes, and tachyon condensation. The notion of stability of bundles has a corresponding physical interpretation (pi-stability $)^{5}$ which is being developed mathematically as well. ${ }^{2,7}$. The surprises which are described in this talk will need to be incorporated into these notions.

Naively, the brane described by $E$ is to be identified with the sheaf $i_{*} E$ of the derived category. But this is not quite correct due to anomalies; 
the standard correspondence between objects of the derived category and branes has to be modified.

Now if two branes $S, T$ are considered, with strings from $S$ to $T$, there are various combinations of Dirichlet and Neumann conditions at the respective ends of the string. The fermion zero modes couple to directions in $S$ and $T$ as well as to directions not in either $S$ nor $T$. Geometrically, these correspond to

$$
T(S \cap T), \quad \mathcal{N}=\frac{T(X)}{T(S) \oplus T(T)}
$$

respectively, where the notational simplification of writing e.g. $T(S)$ in place of $T^{1,0}(S)$ has been adopted. The triviality of

$$
\operatorname{det}(T(S \cap T)) \otimes \operatorname{det}(\mathcal{N})
$$

is required to soak up fermion zero modes. This is equivalent to the triviality of the bundle

$$
\operatorname{det}\left(N_{S \cap T, T}\right) \otimes \operatorname{det}\left(N_{S \cap T, S}\right)
$$

where $N_{S \cap T, T}$ is the bundle of normal vector fields to $S \cap T$ in $T$.

\section{The Freed-Witten anomaly}

An important anomaly in the path integral measure was described by Freed and Witten ${ }^{6}$. There are two interesting factors in the path integral:

$$
\operatorname{Pfaff}(D)
$$

the Pfaffian of the worldsheet Dirac operator, and

$$
\exp \left(i \int_{\partial \Sigma} A\right)
$$

They found that the Pfaffian has an anomaly, characterized by the second Stiefel-Whitney class $w_{2}(S) \in H^{2}\left(S, \mathbf{Z}_{2}\right)$ : that in traversing a family of images of the boundary of the worldsheet $\partial \Sigma$ parametrized by a curve $C$,

$$
\operatorname{Pfaff}(D) \mapsto(-1)^{\left\langle w_{2}(S), C \times \partial \Sigma\right\rangle} \operatorname{Pfaff}(D)
$$

So $\exp \left(i \int_{\partial \Sigma} A\right)$ must transform in the same way to cancel the anomaly:

$$
\exp \left(i \int_{\partial \Sigma} A\right) \mapsto(-1)^{\left\langle w_{2}(S), C \times \partial \Sigma\right\rangle} \exp \left(i \int_{\partial \Sigma} A\right)
$$


This says that the gauge field does not live in a bundle, but rather in a twisted bundle. In fact, the gauge field must live in a twisted bundle $E$ of the form

$$
E=B \otimes(L)^{1 / 2},
$$

where $B$ is an ordinary bundle, and $L$ is a line bundle with

$$
c_{1}(L) \equiv w_{2}(S)(\bmod 2) .
$$

This suggests that the map

$$
\text { Bundles on } S \rightarrow \text { physical branes }
$$

should not be the identity map, but should be modified to

$$
E \mapsto E \otimes L^{1 / 2} .
$$

for some $L$. The line bundle $L$ corresponds to a choice of $\operatorname{Spin}^{c}$ structure on $X$. Any bundle $L$ satisfying (2) will give an identification, but only one choice will identify the open string spectrum with Ext groups of sheaves.

A complex manifold $X$ need not be spin, but is always $\operatorname{Spin}^{c}$. In fact, there is a canonical Spin $^{c}$ structure $^{11}$, and it is not a surprise that this is the one that gives the twisting of the gauge bundle that relates the spectrum to Ext groups. The result is that the correct identification of bundles with twisted sheaves is given by

$$
E \mapsto E \otimes\left(K_{S}^{*}\right)^{1 / 2}
$$

Said differently, this corresponds to a trivialization of a twisting of K-theory dictated by the anomaly.

\section{Computation of the spectrum}

With all the machinery in place, the method for computing the spectrum can now be outlined in the case of parallel coincident branes on $S \hookrightarrow X$. Denote by $E, F$ the gauge bundles on the respective ends of the string. The boundary vertex operators are of the form

$$
b_{\bar{\imath}_{1} \cdots \bar{z}_{n}}^{\alpha \beta j_{m} \cdots j_{m}}\left(\phi_{0}\right) \eta^{\bar{\tau}_{1}} \cdots \eta^{\bar{\imath}_{n}} \theta_{j_{1}} \cdots \theta_{j_{m}} .
$$

Recall the effect of the boundary conditions: the $\theta$ indices are determined by their values in directions normal to $S$, the $\eta$ indices are constrained to only live along directions tangent to $S$, and $\phi$ zero modes $\phi_{0}$ are constrained to only map out $S$. 
By explicit computation ${ }^{9}$, the BRST cohomology classes are in one-toone correspondence with ext groups

$$
\operatorname{Ext}_{X}^{n}\left(i_{*}(E), i_{*} F\right)
$$

In particular, if $E=F$ then $\operatorname{Ext}_{X}^{1}\left(i_{*} E, i_{*} E\right)$ describes first order deformations of $E$ as a sheaf or object of the derived category.

Here is a small part of the computation. Restricting attention to $\theta$ indices tangent to $S$, the BRST condition determines bundle-valued differential forms living on $S$, i.e.

$$
b_{\bar{\imath}_{1} \cdots \bar{\tau}_{n}}^{\alpha \beta j_{1} \cdots j_{m}}\left(\phi_{0}\right) \eta^{\bar{\imath}_{1}} \cdots \eta^{\bar{\imath}_{n}} \theta_{j_{1}} \cdots \theta_{j_{m}} \in H^{n}\left(S, E^{*} \otimes F \otimes \Lambda^{m} N_{S / X}\right)
$$

where $N_{S / X}$ is the normal bundle to $S$ in $X$.

Putting the boundary conditions

$$
\theta_{i}=\left(\operatorname{Tr} F_{i \bar{\jmath}}\right) \eta^{\bar{\jmath}}
$$

back in iteratively, the local to global spectral sequence

$$
E_{2}^{p, q}: H^{p}\left(S, \mathcal{E}^{\vee} \otimes \mathcal{F} \otimes \Lambda^{q} N_{S / X}\right) \Longrightarrow \operatorname{Ext}_{X}^{p+q}\left(i_{*} \mathcal{E}, i_{*} \mathcal{F}\right)
$$

is reproduced exactly, each iteration corresponding to an iteration of the spectral sequence. This shows that the BRST cohomology is the abutment of the spectral sequence, i.e. the anticipated groups $\operatorname{Ext}_{X}^{n}\left(i_{*} \mathcal{E}, i_{*} \mathcal{F}\right)$.

The Freed-Witten anomaly is not manifest here - it cancels out of the opposite ends of the string, so has been suppressed from the notation. But this is not the case in general. Consider branes

$$
\begin{aligned}
& i: S \hookrightarrow X, \text { gauge field in } E \otimes\left(K_{S}^{*}\right)^{1 / 2} \\
& j: T \hookrightarrow X, \text { gauge field in } F \otimes\left(K_{T}^{*}\right)^{1 / 2}
\end{aligned}
$$

Now the BRST cohomology is on one-to-one correspondence with elements of

$$
\left.\operatorname{Ext}_{X}^{n}\left(i_{*} E, j_{*} F\right)\right)
$$

The computation matches the local to global spectral sequence with $E_{2}$ term

$$
\begin{gathered}
E_{2}^{p, q}=H^{p}\left(S \cap T,\left.\left.E^{*}\right|_{S \cap T} \otimes F\right|_{S \cap T} \otimes \Lambda^{q-m} \mathcal{N} \otimes \operatorname{det} N_{S \cap T / T}\right) \\
\Longrightarrow \operatorname{Ext}_{X}^{p+q}\left(i_{*} E, j_{*} F\right)
\end{gathered}
$$


where $m$ is the rank of $N_{S \cap T / T}$ and

$$
\mathcal{N}=\frac{T(X)}{T(S) \oplus T(T)} .
$$

as before. Note that the term $\operatorname{det} N_{S \cap T / T}$ is physically absent if the anomaly is ignored. The computation works only because the B-model anomaly cancels the Freed-Witten anomaly. To see this, use the identity

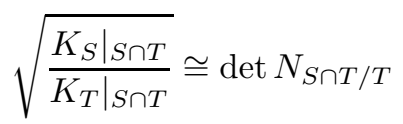

This may seem a bit odd that the B-model anomaly is required, since for physical strings, the condition on the triviality of

$$
\operatorname{det}\left(N_{S \cap T, T}\right) \otimes \operatorname{det}\left(N_{S \cap T, S}\right)
$$

is absent, while there is general confidence that the derived category and ext groups gives the correct answer. This suggests that for physical strings (3) is a new supersymmetry selection rule.

I would like to use this description of the spectrum to compute correlation functions, but there are a number of serious obstacles. All that can be said at present is that three-point functions can be computed via the Yoneda pairing.

\section{Generalizations}

The analysis above extends to a wide range of string models, with similar results. The extension to orbifolds ${ }^{8}$ shows that this analysis is not constrained to large radius and shows how Ext groups in (quotient) stacks arise in string theory. The extension to flat $B$-field backgrounds ${ }^{3}$ shows how Ext groups in the derived category of twisted sheaves arises in string theory. Finally, the boundary conditions can correspond to sheaves on nonreduced schemes, which can sometimes be described physically by turning on nilpotent Higgs fields. The resulting spectrum coincides with Ext groups of sheaves associated to these nonreduced schemes ${ }^{4}$.

\section{CONCLUSIONS}

Open string vertex operators can be explicitly and completely described by complex geometry, in the situation where the boundary conditions correspond to gauge fields in (twisted) bundles on holomorphic submanifolds and beyond. It is desirable to describe the twisting needed to cancel the 
anomaly intrinsically in terms of the derived category. Thus boundary branes can be described directly in CFT. It is desirable to extend existing methods to compute higher correlation functions.

\section{Acknowledgments}

I would like to thank Eric Sharpe for many valuable discussions and for collaboration on this topic, and Robert Karp for comments on an earlier draft.

\section{References}

1. P. Aspinwall and A. Lawrence, Derived categories and zero-brane stability, JHEP 0108004 (2001), hep-th/0104147.

2. T. Bridgeland, Stability conditions on triangulated categories, math. AG/0212237.

3. A. Caldararu, S. Katz, and E. Sharpe, D-branes, B fields, and Ext groups, ATMP 7 382-403 (2003), hep-th/0302099.

4. R. Donagi, S. Katz, and E. Sharpe, Spectra of D-branes with Higgs vevs, hep-th/0309270.

5. M. Douglas, D-branes, categories, and $\mathcal{N}=1$ supersymmetry, J. Math. Phys. 42 2818-2843 (2001), hep-th/0011017.

6. D. Freed and E. Witten, Anomalies in string theory with D-branes, hep-th/9907189.

7. A. Gorodentscev, S. Kuleshov, A. Rudakov, Stability data and t-structures on a triangulated category, math.AG/0312442.

8. S. Katz, T. Pantev, and E. Sharpe, D-branes, orbifolds, and Ext groups, Nucl. Phys. B673 263-300 (2003), hep-th/0212218.

9. S. Katz and E. Sharpe, D-branes, open string vertex operators, and Ext groups, Adv. Theor. Math. Phys. 6 979-1030 (2003), hep-th/0208104.

10. M. Kontsevich, Homological algebra of mirror symmetry, Proceedings of the 1994 International Congress of Mathematicians, Birkäuser, Zürich, 1995, p. 120, alg-geom/9411018.

11. H. B. Lawson and M.-L. Michelson, Spin Geometry, Princeton University Press, Princeton 1989.

12. E. Sharpe, D-branes, derived categories, and Grothendieck groups, Nucl. Phys. B561 433-450 (1999), hep-th/9902116.

13. E. Sharpe, Lectures on D-branes and sheaves, hep-th/0307245.

14. E. Sharpe, Mathematical Aspects of D-branes, these proceedings.

15. E. Witten, Mirror Manifolds and Topological Field Theory, in Essays on Mirror Manifolds, S.T. Yau (ed), International Press, Hong Kong $1 \overline{992 .}$ 